\title{
Laser ablation modelling of aluminium, silver and crystalline silicon for applications in photovoltaic technologies
}

\author{
M. Colina , C. Molpeceres, M. Morales, F. Allens-Perkins, G. Guadaño and \\ J. L. Ocaña
}

Laser material processing is being extensively used in photovoltaic applications for both the fabrication of thin film modules and the enhancement of the crystalline silicon solar cells. The two temperature model for thermal diffusion was numerically solved in this paper. Laser pulses of 1064,532 or $248 \mathrm{~nm}$ with duration of 35,26 or $10 \mathrm{~ns}$ were considered as the thermal source leading to the material ablation. Considering high irradiance levels $\left(10^{8}-10^{9} \mathrm{~W} \mathrm{~cm}^{-2}\right)$, a total absorption of the energy during the ablation process was assumed in the model. The materials analysed in the simulation were aluminium (Al) and silver (Ag), which are commonly used as metallic electrodes in photovoltaic devices. Moreover, thermal diffusion was also simulated for crystalline silicon (c-Si). A similar trend of temperature as a function of depth and time was found for both metals and c-Si regardless of the employed wavelength. For each material, the ablation depth dependence on laser pulse parameters was determined by means of an ablation criterion. Thus, after the laser pulse, the maximum depth for which the total energy stored in the material is equal to the vaporisation enthalpy was considered as the ablation depth. For all cases, the ablation depth increased with the laser pulse fluence and did not exhibit a clear correlation with the radiation wavelength. Finally, the experimental validation of the simulation results was carried out and the ability of the model with the initial hypothesis of total energy absorption to closely fit experimental results was confirmed.

Keywords: Laser ablation, Two temperature model, Thin film photovoltaic technology

\section{Introduction}

Laser processing of materials is being used in a wide range of technological fields as microelectronic and photovoltaic (PV) technologies. In particular, laser ablation processes are required in monolithic interconnection of thin film photovoltaic modules., ${ }^{1,2}$ Moreover, it has been demonstrated that processes involving lasers as a crucial tool, such as laser grooved buried front contact (LGBC) ${ }^{3}$ and laser fired contact (LFC) ${ }^{4}$ improve efficiencies for crystalline silicon photovoltaic devices. Consequently, research in laser processing of the materials used in the photovoltaic industry has become a highly attractive field, especially since laser technology developments have the potential to further improve the performance of photovoltaic devices. ${ }^{5}$

Fabrication processes of thin film photovoltaic modules and crystalline silicon solar cells treated with
LGBC and LFC involve the use of nanosecond lasers, for cell interconnection in the first case, and for the enhancement of the devices in the second case. Femtosecond and picosecond lasers have focused increasing attention in applications as micromachining, as they are known to significantly reduce thermal damage of the treated materials. The industrial use of nanosecond laser in PV can be attributed to different factors, such as relatively recent availability of ultrashort lasers and running and maintenance costs. Nevertheless, all the efforts focused on the achievement of a deeper understanding of both ultrashort and short laser pulse interaction with different materials use in the photovoltaic industry have a marked significance, since it could permit a certain level of control of the different adverse effects that could worsen the electrical properties of the fabricated devices. Laser processing simulation by means of computational models constitutes a very useful tool when considering the implementation of laser stages at industrial scale. Thus, such simulation permits a deeper understanding of physical processes involved and, consequently, it is expected to support the industry in further developing of fabrication steps. In 
this work, the two temperature model for thermal diffusion $^{6}$ was employed to simulate the thermal response of different materials after irradiation with a laser beam having duration in the range of tens of nanoseconds. The two temperature model has been widely employed for metal ablation with ultrashort pulse lasers: $\mathrm{Cu},{ }^{7} \mathrm{Au},{ }^{8,9} \mathrm{Al}^{10,11}$ and $\mathrm{Ag} .{ }^{11}$ Although the present work is focused on nanosecond lasers, the two temperature model implemented is intended to allow further applications to ultrashort laser pulse interaction with materials, especially when it is taken into account that these lasers have started to use at laboratory level in researches for $\mathrm{PV}^{5}$ In order to validate simulation results, experimental tests were also carried out at the laboratory, for which different laser processing conditions in accordance with simulated parameters were analysed. Materials considered for both numerical and experimental studies were bulks of aluminium (Al) and silver (Ag), metals which are commonly used in PV devices as contact electrodes. ${ }^{12}$ Additionally, the thermal response of crystalline silicon (c-Si) samples was also studied, not only because of its inherent interest, but also as a first approximation to further studies involving amorphous silicon (a-Si), this material being used in silicon thin film PV technology as the photosensitive one. Finally, a thermal criterion for the determination of the ablation depth for the different materials under investigation was considered.

\section{Heat diffusion model}

\section{Two temperature model for metals}

In the two temperature model, two different thermal transport subsystems, electrons and lattice, are considered to describe the interaction of an ultrashort laser pulse with a material. Thus, the energy of the incident photons is first rapidly absorbed by the free electrons at the metal surface due to the low heat capacity of the electron subsystem. Consequently, the laser pulse creates a non-equilibrium distribution of very high temperature electrons, leaving the lattice temperature temporally unaffected. Afterwards, the absorbed energy is thermalised within the electron subsystem, with subsequent energy transfer to the lattice. The electron energy is transferred to the lattice subsystem by electron phonon coupling, which leads to electron cooling and lattice heating until thermal equilibrium is reached. According to the subsystem definition, the spatial and time evolutions of the electron and lattice temperatures $\left(T_{\mathrm{e}}\right.$ and $T_{1}$ respectively) are described by the following onedimensional, two temperature diffusion equations, which set the basis for the so called two temperature model.

$$
\begin{aligned}
C_{\mathrm{e}} \frac{\partial T_{\mathrm{e}}}{\partial t} & =-\frac{\partial}{\partial z}\left(\kappa_{\mathrm{e}} \frac{\partial T_{\mathrm{e}}}{\partial z}\right)-g\left(T_{\mathrm{e}}-T_{1}\right)+S(t) \\
C_{1} \frac{\partial T_{1}}{\partial t} & =g\left(T_{\mathrm{e}}-T_{1}\right)
\end{aligned}
$$

where $z$ is the direction perpendicular to the plane of the sample, $t$ is the time from the initiation of the pulse, $S$ is the laser heating source term, $C_{\mathrm{e}}$ and $C_{1}$ are the heat capacities (per unit volume) for the electron and the lattice subsystems respectively, $g$ is the parameter characterising the electron lattice coupling and $\kappa_{\mathrm{e}}$ is the heat conductivity for the electron subsystem. It is important to note that the electronic heat capacity $C_{\mathrm{e}}$ depends on the temperature and for metals can be written as follows

$$
C_{\mathrm{e}}=\frac{\pi^{2} n_{\mathrm{e}}}{2}\left(\frac{k_{\mathrm{B}} T_{\mathrm{e}}}{\varepsilon_{\mathrm{f}}}\right) k_{\mathrm{B}} \equiv \gamma T_{\mathrm{e}}
$$

where $\gamma$ is called electronic heat capacity constant, $n_{\mathrm{e}}$ is the density of free electrons, $k_{\mathrm{B}}$ is the Boltzmann constant and $\varepsilon_{\mathrm{f}}$ is the Fermi energy. Temperature dependency of the heat conductivity $\left(\kappa_{\mathrm{e}}\right)$ has not been considered in our calculations.

The absence of the heat flux term in equation (2) is due to the slow heat diffusion in the lattice subsystem. Finally, the laser source term, $S$, can be written as

$$
S=\frac{0.94 \alpha I_{0}}{\tau} A \exp \left[-(4 \ln 2)\left(\frac{t-\tau}{\tau}\right)-\alpha z\right]
$$

where $\tau$ is the pulse duration, $F$ is the incident laser intensity, $\alpha$ is the absorption coefficient and the absorptivity, $A$, is given by $1-R$, where $R$ is the reflectivity. The later is generally close to 1 for metals, but it can drop to values as low as $0 \cdot 1$ during the laser ablation process, when the irradiance is high enough $\left(\sim 10^{8} \mathrm{Wcm}^{-2}\right){ }^{13}$ This decrease in the reflectivity occurs in time scales rather smaller (tens of picoseconds) than the pulse duration (tens of nanoseconds). ${ }^{14}$ The sudden increase in the material temperature could cause a roughening of the surface and a subsequent increase of the absorptivity. On the other hand, for range of laser fluences considered in this work $\left(1-100 \mathrm{~J} \mathrm{~cm}^{-2}\right)$ where irradiances of approximately $10^{8}-10^{9} \mathrm{~W} \mathrm{~cm}^{-2}$ take place, the absorption of laser generated vapour plasma should be also taken into account. ${ }^{15}$ As a first approximation in order to consider the combination of this two effects we are assuming total absorption in our study $(A=1, R=0)$.

For solving the diffusion equations, the same temperature before the interaction with the laser pulse was assumed for both electron and lattice subsystems so that the initial conditions were given by $T_{\mathrm{e}}(x, t=0)=$ $T_{1}(x, t=0)=T_{\mathrm{o}}=300 \mathrm{~K}$. Finally, boundary conditions were defined as follows

$$
\left.\frac{\partial T_{\mathrm{e}}}{\partial z}\right|_{\mathrm{Z}=0}=\left.\frac{\partial T_{\mathrm{e}}}{\partial z}\right|_{\mathrm{Z}=\mathrm{L}}=0
$$

The running time considered for equations solving was 3000 ns. Regarding the spatial distribution, the implemented numerical model consists of 360 spatial points distributed on a logarithmic scale, as the spatial variation in the electron temperature in the outermost layer is much larger than further into the bulk. Finally, the maximum depth was set as $30 \mu \mathrm{m}$.

Once the temperature evolution is calculated, an ablation criterion can be established. Thus, after the laser pulse, the maximum depth for which the total energy stored in the material is equal to the vaporisation enthalpy is considered as the ablation depth. Accounting for the combined energy in the electron and lattice subsystems, the applied ablation criterion is ${ }^{11}$

$$
C_{1} T_{1}+\left(\frac{\gamma T_{\mathrm{e}}^{2}}{2}\right) \geqslant \rho \Omega_{\mathrm{vap}}
$$

where $\rho$ is the density and $\Omega_{\text {vap }}$ is the mass specific 
enthalpy of vaporisation. Consequently, the ablation depth is, in the present model, the maximum depth where the inequality is fulfilled at any time during the simulation.

It is worth mentioning that for nanosecond pulses duration as those employed in the present work the two temperature model can be simplified as the temperatures corresponding to both subsystems become equal. In this case, typical lattice heat propagation equation with a single characteristic temperature can be applied. In any case, the two temperature formalism has been maintained throughout this work considering further application to short pulse durations, for which the two temperature model is required due to different temperature evolution in both subsystems.

\section{Heat diffusion model for crystalline silicon}

The dependence of the electronic heat capacity constant $(\gamma)$ on the free carrier density makes it difficult to establish a value for $\gamma$ in semiconductor materials, such as crystalline silicon (c-Si). In this case, the free carrier density varies over a large range under non-equilibrium conditions due to different effects such as electron hole pair creation, Auger recombination and impact ionisation. ${ }^{16}$ Consequently, the two temperature model involving the electron and lattice subsystems can not be applied in a straightforward manner. Therefore, a simplified approach was considered for the study of c$\mathrm{Si}$, in which only heat diffusion on the lattice system was taken into account, that being an acceptable approximation for nanosecond pulses. ${ }^{17}$ Thus, the distribution of the temperature was calculated according to equation (1) making $T_{\mathrm{e}}=T_{1}=T$. Similarly, the determination of the temperature evolution into the material, made it possible to introduce a criterion for the calculation of the ablation depth. In order to do this, the term $\gamma$ in the equation (6) was neglected and the ablation criterion was applied again for $\mathrm{c}-\mathrm{Si}$.

\section{Experimental setup}

\section{Laser systems and samples}

The employed experimental system involved two different lasers. The first one was a $\mathrm{Nd}: \mathrm{YVO}_{4}$ pulsed DPSS laser (Spectra Physics), which was used in its two available wavelengths: 532 and $1064 \mathrm{~nm}$ and with pulse durations of 10 and $26 \mathrm{~ns}$ respectively. The second laser system was a ND:YAG pulsed DPSS laser (Trumpf VectorMarck Workstation VWS 800) with a wavelength of $1064 \mathrm{~nm}$ and $35 \mathrm{~ns}$ of pulse width.

In order to carry out the experimental validation of the developed numerical simulation, two sheets of metals were processed with the laser systems described above. The metals studied were aluminium (Al) and silver (Ag), both with a thickness of $0.125 \mathrm{~mm}$ and with an average roughness of $0.7 \mu \mathrm{m}$. An $\mathrm{n}$-doped silicon wafer of $300 \mu \mathrm{m}$ thickness and an average roughness of $0.2 \mu \mathrm{m}$ was also subjected to study. On the one hand, the metals and the silicon wafer were first processed with the $532 \mathrm{~nm}$ laser and $10 \mathrm{~ns}$ pulse duration. On the other hand, processing with $1064 \mathrm{~nm}$ wavelength was performed with pulse durations of 35 and $26 \mathrm{~ns}$ for $\mathrm{Al}$ and Ag respectively.

The experimental tests consisted in performing separated laser shots, each one corresponding to a single laser pulse onto the materials. For each experiment, the material surface was placed approximately on the focus of the laser beam focalisation system, obtaining a beam radius of about $35 \mu \mathrm{m}$. In order to modify the fluence for each pulse, the laser pulse energy was varied, while maintaining the beam diameter invariable. For each pulse, the maximum fluence was calculated as follows

$$
F=\frac{2 E_{\mathrm{p}}}{\pi \omega^{2}}
$$

where $E_{\mathrm{p}}$ is the energy of the laser pulse and $\omega$ corresponds to the laser beam radius. In order to perform the comparison between numerical and experimental data, this maximum fluence was taken as the term of laser intensity $\left(I_{0}\right)$ included in equation (4).

\section{Measurements and characterisation techniques}

Ablation profile measurements and morphological characterisation have been made using a Confocal Laser Scanning Microscope (CLSM) Leica ICM 1000. The equipment operating wavelength $(635 \mathrm{~nm}$ corresponding to diode laser emission) and the objective numerical aperture and pinhole diameter give the final resolution in axial direction. In confocal microscopy techniques, three-dimensional images are obtained by moving the focus plane and acquiring single images (optical slices) that can be put together building up a three dimensional stack of images that can be digitally processed.

The lateral resolution of the CLSM for step measurements is about $20 \mathrm{~nm}$, which corresponds to theoretical calculations according to the objective specifications. However, global accuracy in $\mathrm{Z}$ measurements is strongly dependent on sample optical reflectivity. Anyway, higher reflectivity gives place to better step measurement accuracy, so the previous value can be considered a reasonable approach taking into account optical behaviour of the studied materials. Despite this high resolution availability in $Z$ measurements, the obtained morphological profiles from spots with ablation depths below $1 \mu \mathrm{m}$, which are typically obtained with fluences close to the ablation threshold, were not employed as experimental data in the ablation curves. In these cases, the shape of the spots could lead to mistakes in the identification of the actual ablation depth due to effects such as irregular distribution of the resolidified material.

\section{Models results}

The two temperature model has been implemented in MatLab $^{18}$ using pdepe function ${ }^{19}$ to solve the system of equations (1) and (2). Numerical results of both metals and crystalline silicon are described below. The evolution of the temperatures is given and the corresponding ablation depths are presented. Simulation parameters used in the model for each material are shown in Table 1, where the optical absorption coefficient for the different wavelengths analysed is also presented.

\section{Metals: $\mathbf{A l}$ and $\mathbf{A g}$}

The evolution of the temperatures $\left(T_{\mathrm{e}}\right.$ and $\left.T_{1}\right)$ as a function of depth and time was analysed for $\mathrm{Al}$ and $\mathrm{Ag}$ at three wavelengths $(248,532$ and $1064 \mathrm{~nm})$. A similar temperature trend was found for both metals regardless of the wavelength employed. In particular, and as already expected, the electron and lattice temperatures 


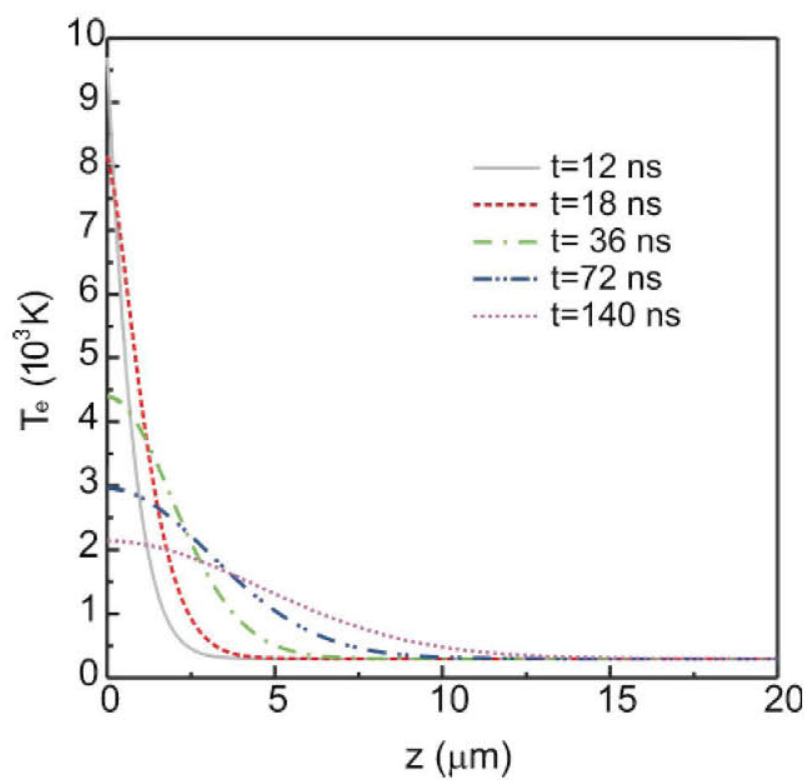

1 Temperature evolution versus material depth at different times: numerical results for Al treated with laser pulse of $532 \mathrm{~nm}, 10 \mathrm{~ns}$ of duration and with maximum fluence of Gaussian beam of $3.8 \mathrm{~J} \mathrm{~cm}^{-2}$

exhibited exactly the same behaviour, this being a consequence of the interaction time regime (tens of nanoseconds). Therefore, only one temperature is required to fully characterise the heat propagation into the material.

In order to show the obtained trend, results corresponding to $\mathrm{Al}$ treated with a laser pulse of $532 \mathrm{~nm}$, $10 \mathrm{~ns}$ pulse duration and fluence of $3.8 \mathrm{~J} \mathrm{~cm}^{-2}$ are presented. Figure 1 shows the evolution of the temperature as a function of depth into the material for different times. A first general trend can be observed, with temperature decreasing as the material depth increases. Besides, this trend is especially sharp just after the pulse. As time increases, the curve is widened, as energy is transferred from the surface to the material bulk.

Figure 2 shows the temperature evolution as a function of time for different material depths. Four different behaviours attending to the studied depth can be distinguished. For the material surface and up to $1 \mu \mathrm{m}$ depth, temperature increases to maximum values of $9500 \mathrm{~K}$ (for $z=0 \mu \mathrm{m}$ ) and $4500 \mathrm{~K}$ (for $z=1 \mu \mathrm{m}$ ) shortly after the pulse when the Gaussian beam reaches its maximum intensity. Afterwards, temperature decreases to values of about $2000 \mathrm{~K}$ for both depths. The second behaviour corresponds to that observed for $3 \mu \mathrm{m}$ of depth, where the maximum value of temperature $(1960 \mathrm{~K})$ is reached later $(60 \mathrm{~ns})$ and the decreasing from this value is less pronounced. Additionally, for depths farther away from the surface $(10 \mu \mathrm{m})$, the temperature begins to increase from times of about $70 \mathrm{~ns}$. Finally, for positions deep into the material $(20 \mu \mathrm{m})$, the variation on the temperature does not achieve the $20 \mathrm{~K}$ for the considered simulation time.

Once the temperature evolution was estimated, the ablation criterion was used for the calculation of the ablation depth. Figure 3 shows the ablation depth obtained for $\mathrm{Al}$ and $\mathrm{Ag}$ as a function of the fluence, $\mathrm{F}$, for all the analysed wavelengths ( $\tau=10 \mathrm{~ns}$ ). For all cases, the ablation depth increases with the fluence. As 


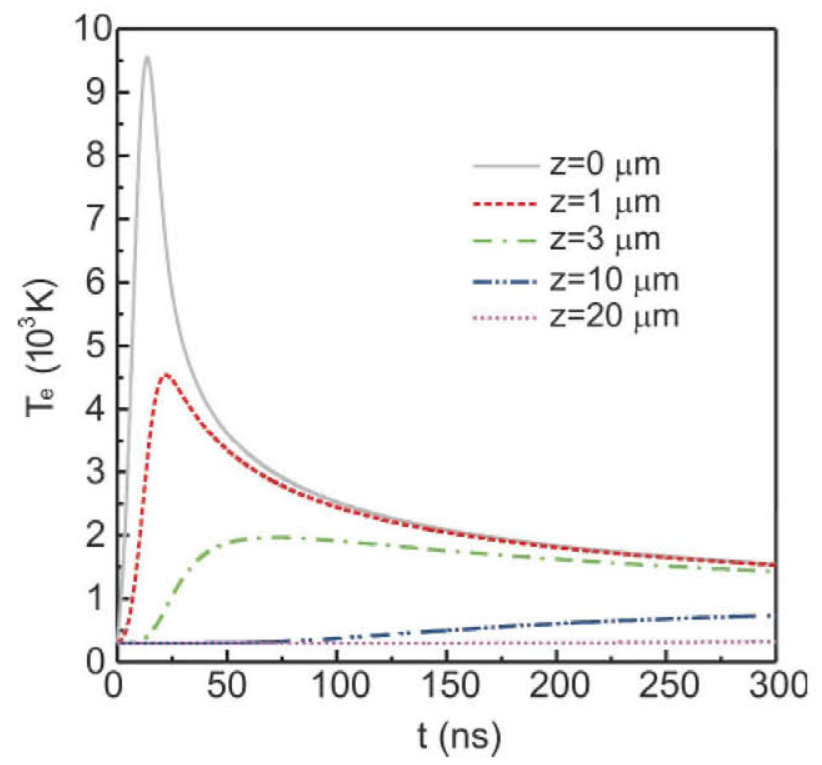

2 Temperature evolution versus time for different material depths: numerical results for Al treated with laser pulse of $532 \mathrm{~nm}, 10 \mathrm{~ns}$ of duration and with maximum fluence of Gaussian beam of $3.8 \mathrm{~J} \mathrm{~cm}^{-2}$
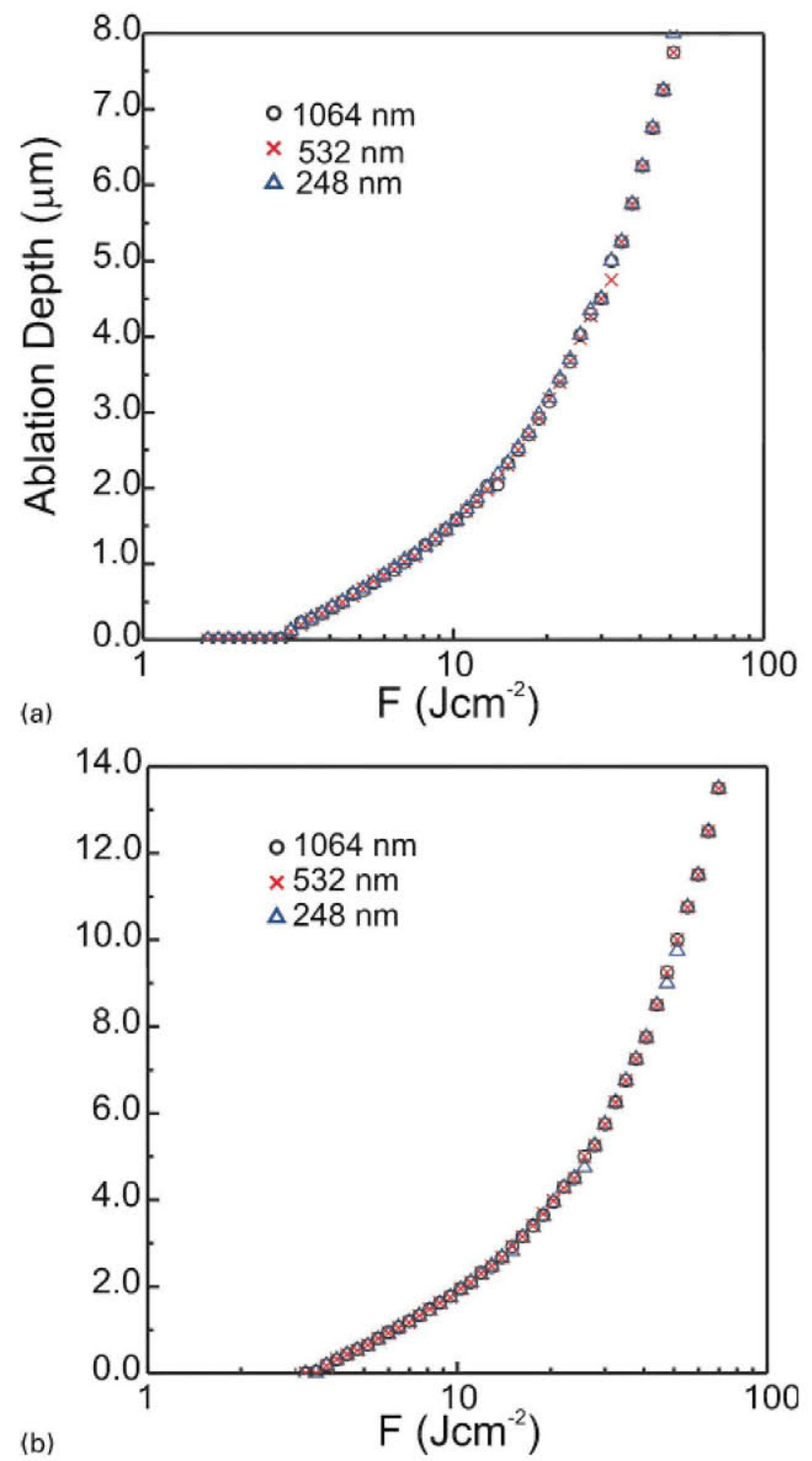

3 Ablation depth versus fluence at different wavelengths for $a \mathrm{Al}$ and $b \mathrm{Ag}(\tau=10 \mathrm{~ns})$

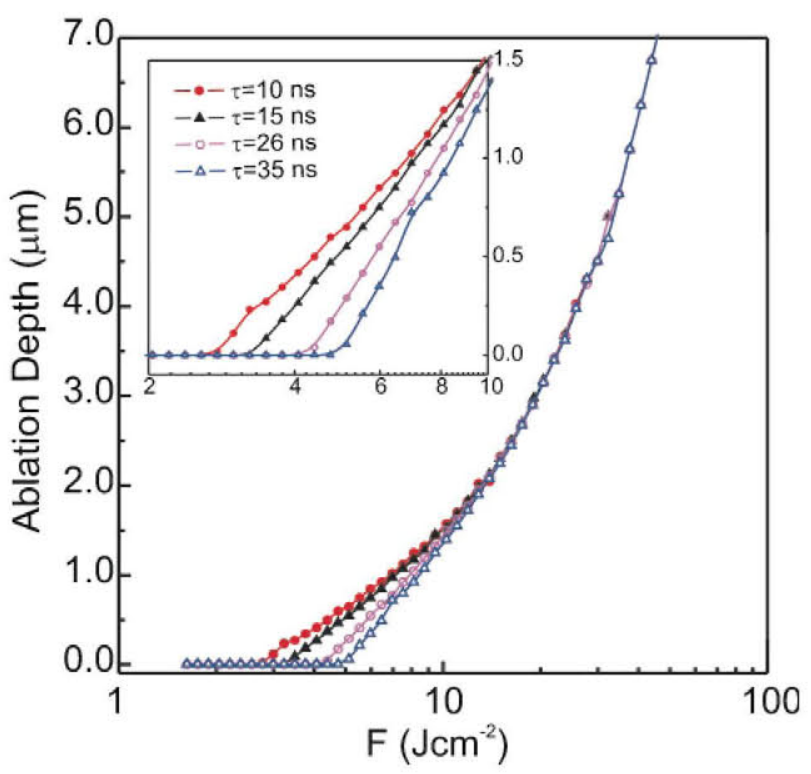

4 Al Ablation depth versus fluence obtained for different pulse duration $(\lambda=1064 \mathrm{~nm})$

expected from the results on temperature evolution, the obtained ablation depth does not exhibit a clear correlation with the radiation wavelength. In addition, threshold values of 2.8 and $3.5 \mathrm{~J} \mathrm{~cm}^{-2}$ for $\mathrm{Al}$ and $\mathrm{Ag}$ respectively were obtained. This result could be interpreted as a consequence of the difference in the thermal conductivity of both materials $\left(\kappa_{\mathrm{e}}=419 \mathrm{~J} \mathrm{~m}^{-1} \mathrm{~s}^{-1} \mathrm{~K}^{-1}\right.$ for $\mathrm{Ag}$ and $\kappa_{\mathrm{e}}=210 \mathrm{~J} \mathrm{~m}^{-1} \mathrm{~s}^{-1} \mathrm{~K}^{-1}$ for $\mathrm{Al}$ ). Likewise, numerical results showed that, once the fluence threshold is reached, the highest ablation depths were obtained for $\mathrm{Ag}$. Indeed, from certain fluence $\left(F=5.3 \mathrm{~J} \mathrm{~cm}^{-2}\right)$ to the maximum energy density studied, the ablation depth obtained for Ag was always higher than that obtained for Al. This result could be also related to the different thermal conductivity of both materials.

The influence of the laser pulse duration on the ablation depth was also evaluated (Fig. 4). In this case, results corresponding to the analysis of $\mathrm{Al}$ irradiated with $1064 \mathrm{~nm}$ are presented. A similar behaviour was also obtained for $\mathrm{Ag}$. In the low fluence regime, the four curves corresponding to the four pulse duration studied were clearly separated. The lowest ablation threshold was obtained for the lowest pulse duration ( $\tau=10 \mathrm{~ns}$ ). As the pulse duration increased, this threshold value for the fluence also increased. As the fluence increases, the curves obtained for the different pulse durations converge to similar values.

An experimental validation of the simulation results was also carried out. In this validation, single laser pulses, each one with a different fluence, were impacted on both $\mathrm{Al}$ and $\mathrm{Ag}$ samples. For $\mathrm{Al}$ treated at $1064 \mathrm{~nm}$, the fluence was varied in the range from 5 to $45 \mathrm{~J} \mathrm{~cm}^{-2}$, whereas a range from 8 to $14 \mathrm{~J} \mathrm{~cm}^{-2}$ was selected for $\mathrm{Ag}$ irradiated at $1064 \mathrm{~nm}$. On the other hand, for $532 \mathrm{~nm}$, the range was varied between 7 and $40 \mathrm{~J} \mathrm{~cm}^{-2}$ for the two metals under study.

Morphological profiles of three spots performed with a laser pulse of $35 \mathrm{~ns}$ of duration and $1064 \mathrm{~nm}$ wavelength onto the sheet of $\mathrm{Al}$ are presented in Fig. 5. As it was mentioned, each spot corresponds to a shot carried out with a single pulse which has a different $F$. The maximum value of the depth found in 


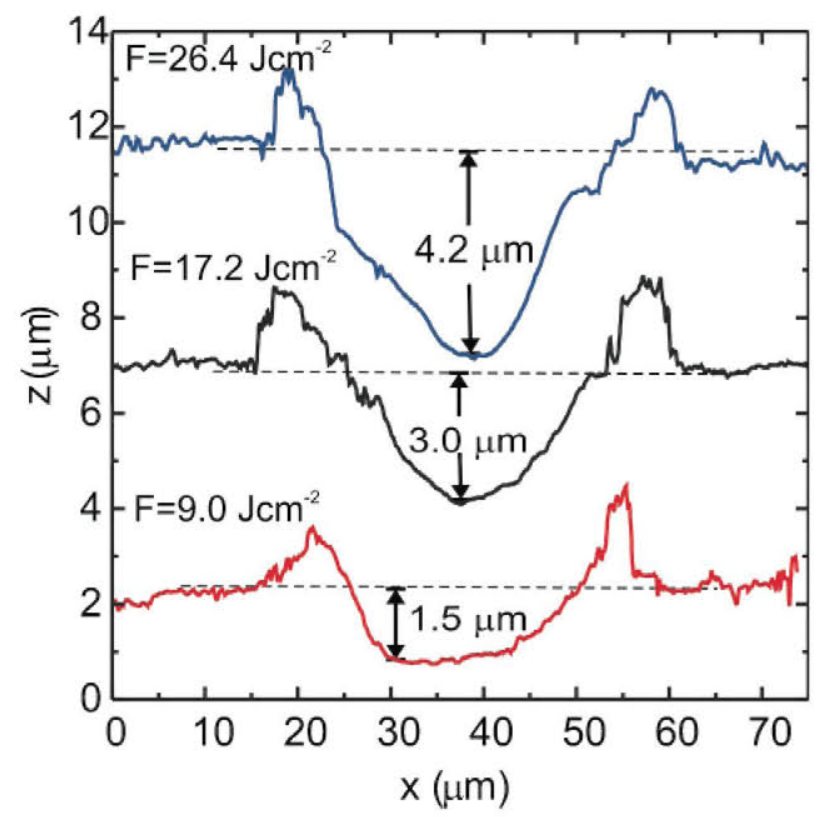

5 CLSM profiles of Al spots obtained at different fluences ( $\lambda=1064 \mathrm{~nm}, \tau=35 \mathrm{~ns}$ )

the morphological profile was chosen as the ablation depth for each spot. As expected, a larger ablation depth is achieved at increasing fluences.

Figure 6 shows a comparative graph between the model results and the experimental points for the $\mathrm{Al}$ sample treated with a laser pulse of $1064 \mathrm{~nm}$ and $35 \mathrm{~ns}$ of duration. Ablation depths obtained from the profiles shown in Fig. 5, are also included in the comparative graph. As can be seen in Fig. 6, both experimental and numerical results are in good agreement. Only for the lowest fluences a certain discrepancy is observed, although it does not exceed the $30 \%$ range.

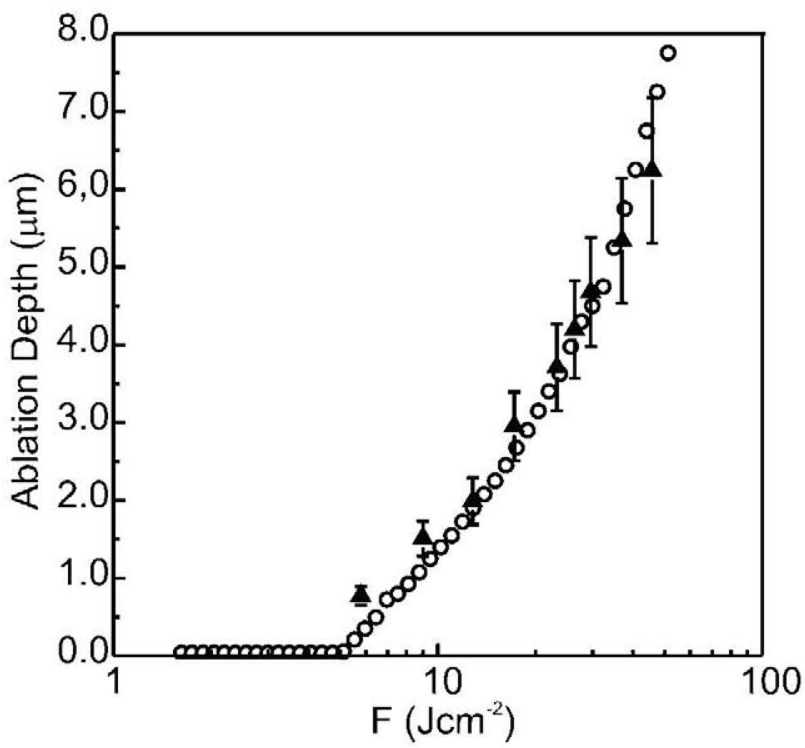

6 Al ablation depth versus fluence: 0 numerical and experimental results $(\lambda=1064 \mathrm{~nm}$ and $\tau=35 \mathrm{~ns})$

Figure 7 shows a comparative graph between the numerical results and the experimental data for the $\mathrm{Ag}$ treated with a laser pulse of $1064 \mathrm{~nm}$ and $26 \mathrm{~ns}$ of duration. Laboratory tests were performed only in the lower fluence range due to experimental limitations regarding beam focalisation. The inset shows the morphological profiles of selected spots. A remarkably good agreement can be observed between the experimental results and the simulated ones (discrepancies do not exceed $15 \%$ ).

Regarding results obtained with laser pulses of $532 \mathrm{~nm}$, Fig. $8 a$ and $8 b$ shows experimental and model results for $\mathrm{Al}$ and $\mathrm{Ag}$ respectively. Both metals were processed with a laser pulse of $532 \mathrm{~nm}$ and $10 \mathrm{~ns}$ of

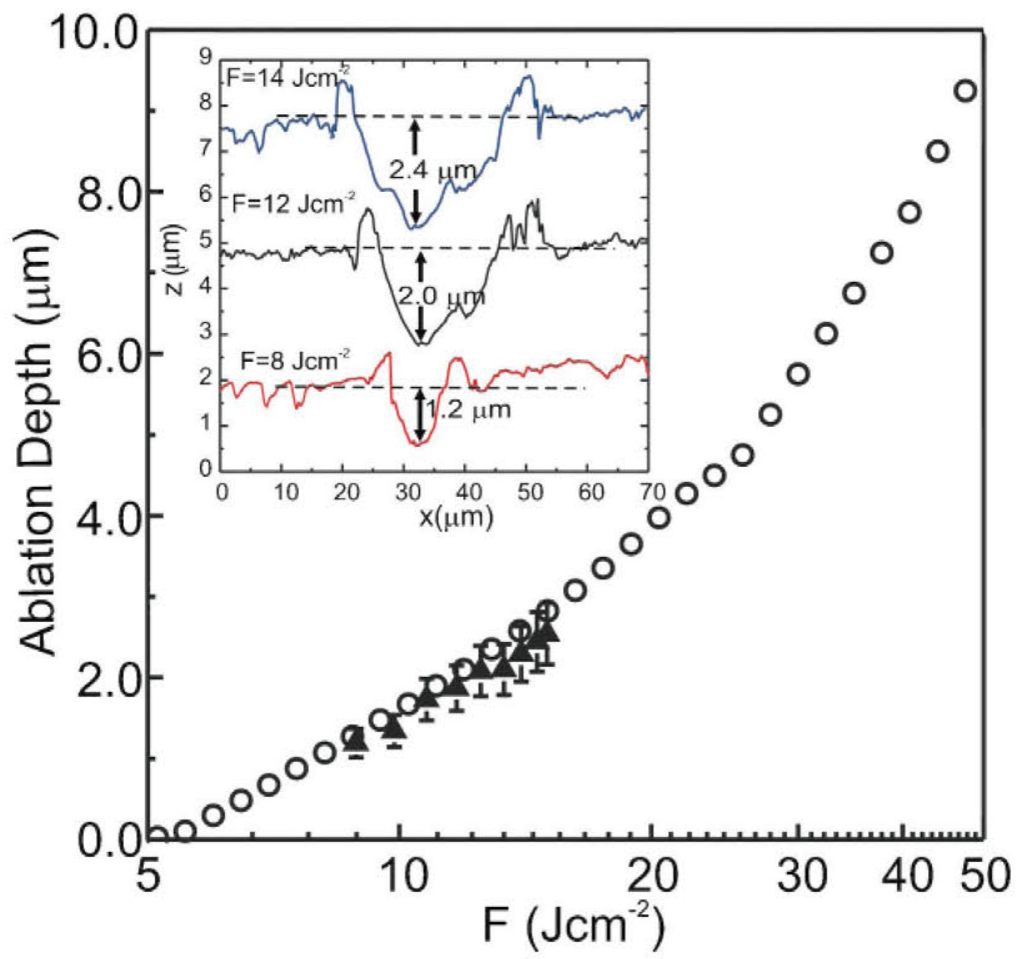

$7 \mathrm{Ag}$ ablation depth versus fluence: $\bigcirc$ numerical and $\Delta$ experimental results ( $\lambda=1064 \mathrm{~nm}$ and $\tau=26 \mathrm{~ns}$ ); CLSM morphological profiles of some spots are also shown (inset) 


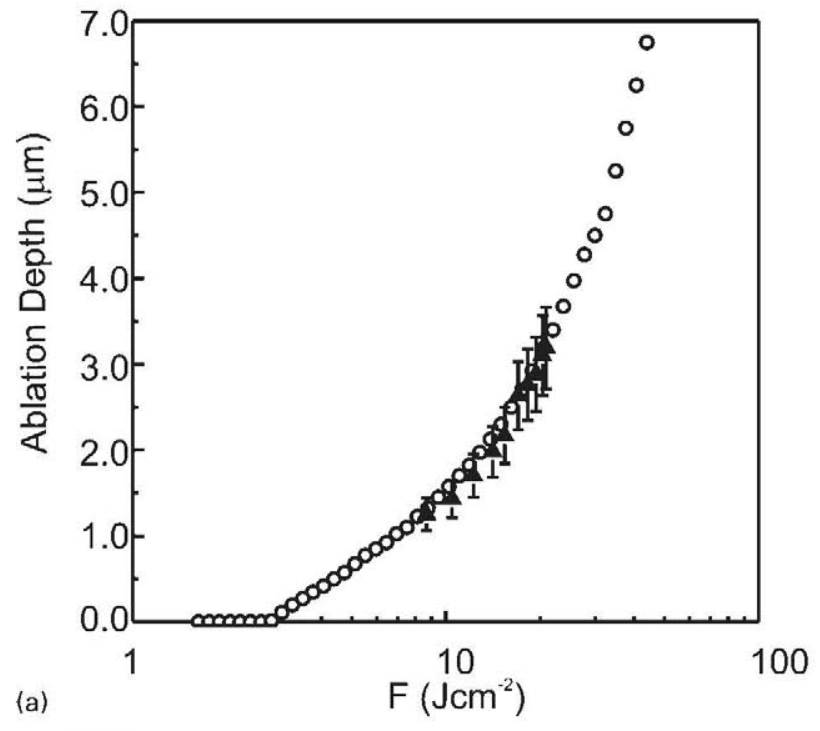

(a)

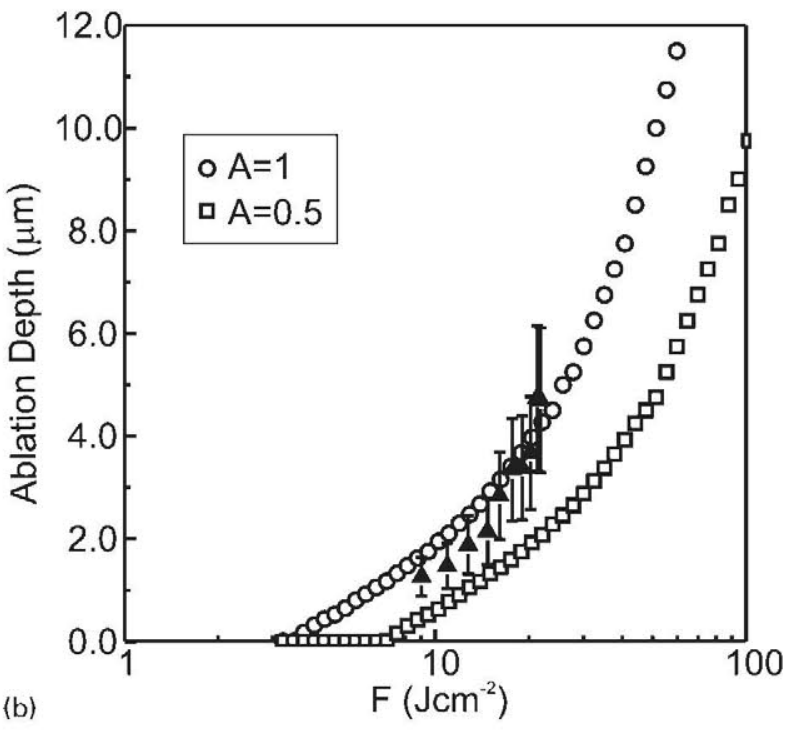

a $\mathrm{Al}$ and $b \mathrm{Ag}$ : $\bigcirc$ numerical $(A=1)$ and $\boldsymbol{\Delta}$ experimental results $(\lambda=532 \mathrm{~nm}$ and $\tau=10 \mathrm{~ns}) ; \square$ numerical results for Ag considering absorptivity of 0.5

8 Ablation depth versus fluence for metals analysed

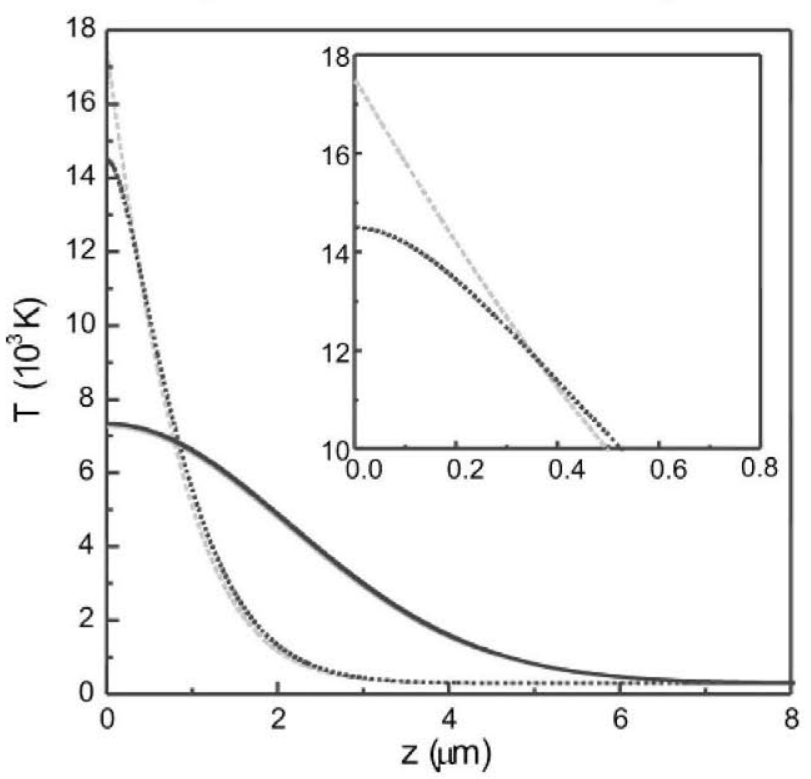

9 Evolution of silicon temperature as function of depth at two times ( $t=12 \mathrm{~ns}$ segmented lines, $t=72 \mathrm{~ns}$ continuous lines) and for two different wavelengths of irradiation ( $\lambda=248 \mathrm{~nm}$ grey colour, $\lambda=532 \mathrm{~nm}$ black colour); pulse conditions $\tau=10 \mathrm{~ns}, F=3.2 \mathrm{~J} \mathrm{~cm}^{-2}$

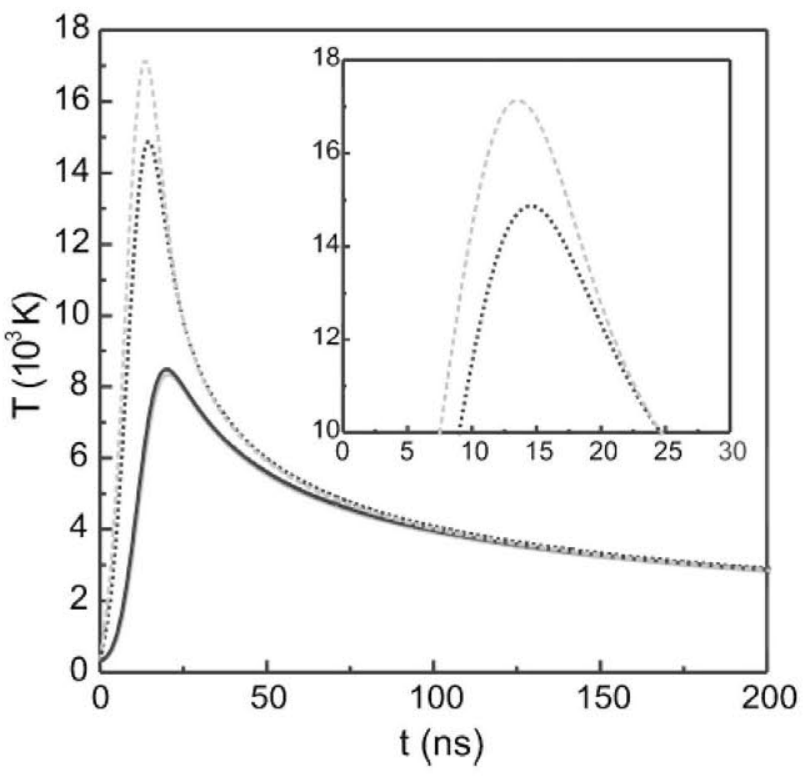

10 Evolution of silicon temperature as function of time at two times ( $t=12$ ns segmented lines, $t=72$ ns continuous lines) and for two different wavelengths of irradiation ( $\lambda=248 \mathrm{~nm}$ grey colour, $\lambda=532 \mathrm{~nm}$ black colour): pulse conditions $\tau=10 \mathrm{~ns}, F=3.2 \mathrm{~J} \mathrm{~cm}^{-2}$

duration. Both graphs show a good agreement between the experiments and the model with slight differences that do not exceed the $15 \%$ range. As it was repeatedly mentioned, for our simulations total absorption $(A=1)$ during the ablation process was assumed. In order to evaluate the effect of the absorptivity on the ablated depth, a value of 0.5 for the absorptivity was considered. Simulation results for $\mathrm{Ag}$ treated with a laser pulse of $532 \mathrm{~nm}$ and $10 \mathrm{~ns}$ of duration and considering an absorptivity of $0 \cdot 5$ are included in Fig. $8 b$. The ablation depths obtained with this value of absorptivity are rather smaller than those obtained for $A=1$ over the whole range of the studied fluences. As can be seen in Fig. $8 b$, experimental results appear to fit considerably better with those model results where total absorption was considered, especially the higher values of fluences. Thus, it can be concluded that the initial assumption of

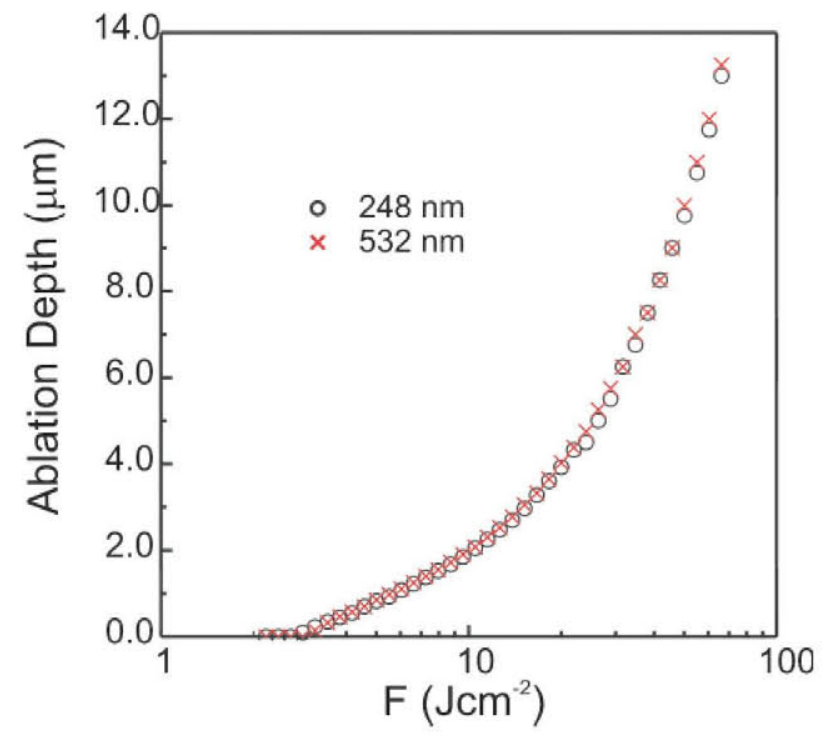

11 Ablation depth versus fluence at different wavelengths for c-Si $(\tau=10 \mathrm{~ns})$ 

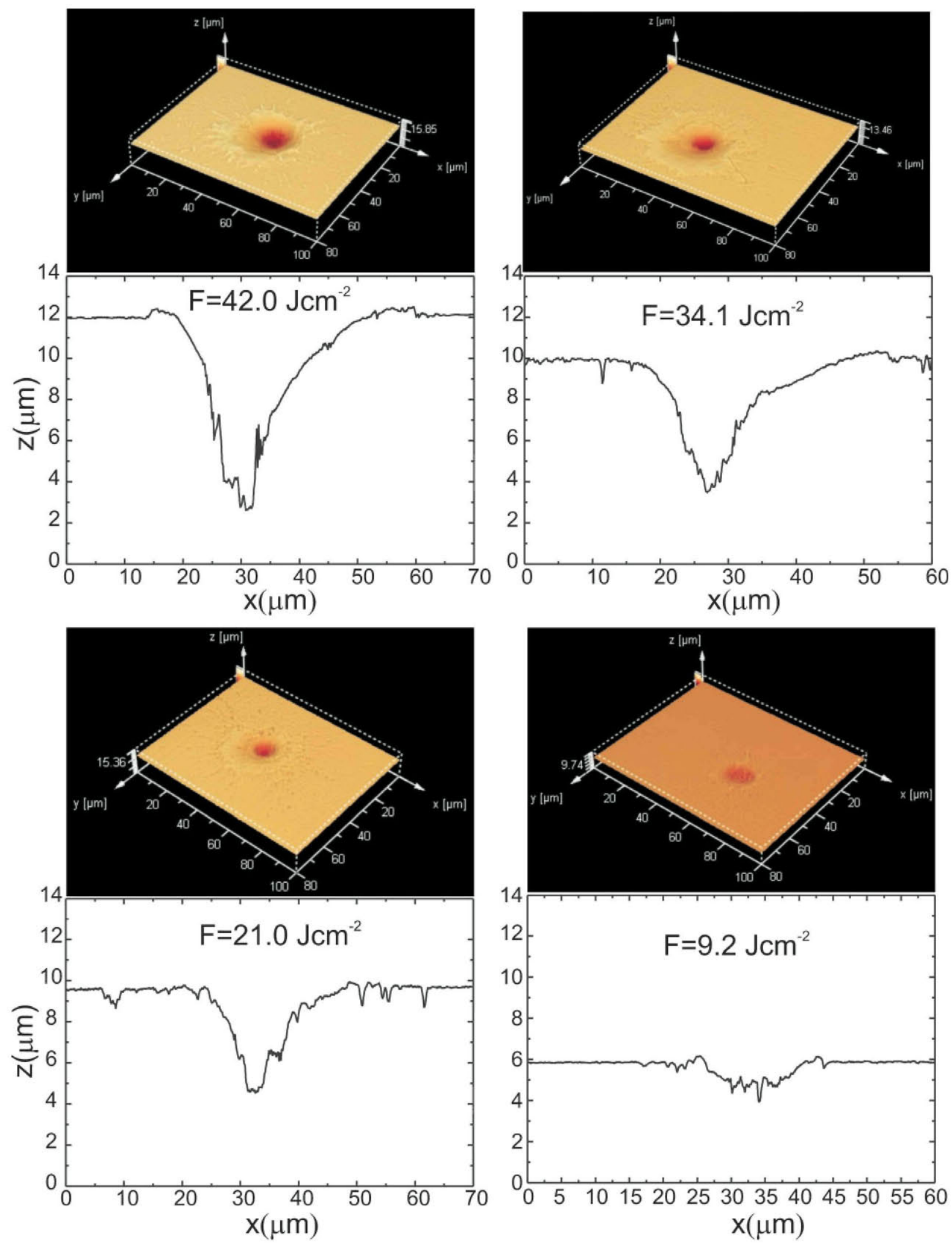

CLSM morphological images and profiles of c-Si processed with laser pulse of $532 \mathrm{~nm}$ and $10 \mathrm{~ns}$ of duration

total energy absorption is, precisely, the more appropriated hypothesis considering the irradiance levels employed in these experiments.

\section{Crystalline silicon}

As indicated in the section on 'Heat diffusion model for crystalline silicon', a simplified approach was assumed for the analysis of the thermal response of crystalline silicon. In this analysis, a numerical solution of the lattice heat conduction equation was first obtained. Once known the temperature at any point in the material, during and subsequent to the laser irradiation, the corresponding ablation depth was calculated. 


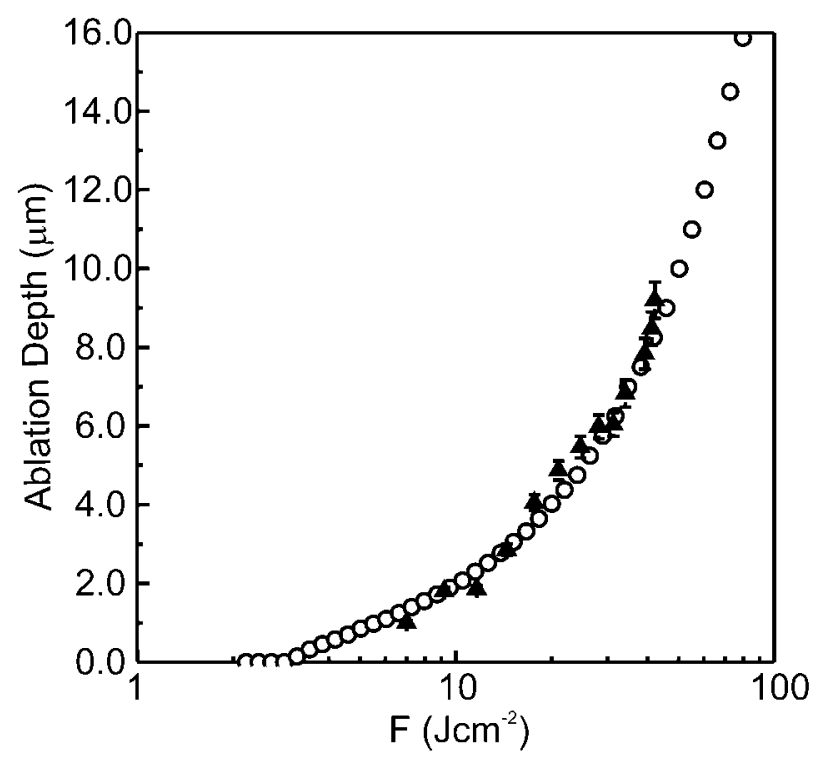

13 c-Si ablation depth versus fluence: $\quad$ Numerical and $\Delta$ experimental results ( $\lambda=532 \mathrm{~nm}$ and $\tau=10 \mathrm{~ns}$ )

The evolution of the silicon temperature as a function of depth at two different times is shown in Fig. 9. In this case, results corresponding to two irradiation wavelengths (532 and $248 \mathrm{~nm}$ ) are represented. Comparing the two curves obtained for each one of the times analysed (12 and $72 \mathrm{~ns}$ ); only a slight difference can be appreciated in those curves corresponding to $12 \mathrm{~ns}$. In this particular case, the material surface reaches a higher temperature for that wavelength $(248 \mathrm{~nm})$ for which the absorption coefficient is higher (Table 1).

Figure 10 shows the temperature evolution of the silicon as a function of time at two fixed positions ( 0 and $1 \mu \mathrm{m})$ and for two different wavelengths irradiation. The maximum temperature acquired on the surface was slightly higher for $248 \mathrm{~nm}$, which exhibits a larger absorption coefficient. Finally, the ablation criterion was used for the calculation of the ablation depth. Figure 11 shows the ablation depth obtained for $\mathrm{c}-\mathrm{Si}$ as a function of the fluence, $F$. As shown, the ablation depth increases with the fluence with a threshold value of $3 \cdot 2 \mathrm{~J} \mathrm{~cm}^{-2}$ for both wavelengths considered.

An experimental validation of the numerical results for crystalline silicon was also carried out. This validation was performed with a wavelength of $532 \mathrm{~nm}$ and pulse duration of $10 \mathrm{~ns}$. The fluence was varied from a value close to the ablation threshold to about $50 \mathrm{~J} \mathrm{~cm}^{-2}$

Figure 12 shows CLSM topographical images of some spots, each one corresponding to a single laser pulse onto the silicon sample and carried out with a different fluence. The CLSM morphological profile of each spot is also shown. As observed from both the morphological profiles and the topographical images, as the fluence decreases, the spot diameter and depth also decreases. A significant thermal damage at the borders of the spots is clearly seen for the highest fluences. Such damage is reduced with the decreasing of the fluence.

Figure 13 shows numerical and experimental results corresponding to crystalline silicon processed with a laser pulse of $532 \mathrm{~nm}$ and $10 \mathrm{~ns}$ of duration. Spots previously shown in Fig. 12 correspond to some of the experimental points represented in this comparative graph. The ability of the simulation to closely fit experimental data is observed. Indeed, discrepancies do not exceed $15 \%$ over the range under study.

\section{Conclusion}

The two temperature model for thermal diffusion has been implemented to study the interaction of some metals, commonly used in photovoltaics ( $\mathrm{Al}$ and $\mathrm{Ag}$ ), with laser pulses of duration in the range of tens of nanoseconds. In this case, temperatures of the lattice and electron subsystems have the same values due to the long time scales of the process. In addition, thermal diffusion on c-Si samples was also analysed.

A thermal ablation criterion was defined for the determination of the ablation depth as a function of the pulse fluence. The calculated ablation depth was observed to increase at increasing fluences for all the materials under study. In addition, an ablation threshold value was determined for each material.

Numerical results regarding the temperature evolution for all the materials and wavelengths studied suggest that differences in the material absorption coefficient for the range analysed do not induce significant changes in the obtained ablation depths. On the other hand, numerical results showed that the variation of the pulse duration has influence on the ablation depth only for low fluences.

Finally, experimental tests were carried out to validate simulation model. Experimental and numerical results for all the analysed materials were in good agreement, thus giving evidence of the suitability of the model employed for the materials and conditions analysed.

\section{Acknowledgement}

This work has been financed by the Spanish National R\&D Plan under CLASICO ENE2007-67742-C04 and PSE-MICROSIL08.

\section{References}

1. S. Hass, A. Gordijn and H. Stiebig: Prog. Photovolt, Res. Appl., 2008, 16, 195-203.

2. K. Yamamoto, A. Nakajima, M. Yoshimoto, T. Sawada, T. Suezaki, M. Ichikawa, Y. Koi, M. Goto, T. Meguro, T. Matsuda, M. Masaraka, T. Sasaki and Y. Tawada: Solar Energ., 2004, 77, 939-949.

3. O. Schultz, S. W. Glunz, W. Warta, R. Preu, A. Grohe, M. Köber, G. P. Willeke, R. Russel, J. Fernandez, C. Morilla, R. Bueno and I. Vincuería: Proc. 21st Eur. Photovolt. Solar Energ. Conf. I, 2006, 826, 521-523.

4. E. Schneiderlöchner, R. Preu, R. Lüdemann and S. W. Glunz: Prog. Photovol., 2002, 10, 29-34.

5. J. Hermann, M. Benfarah, S. Bruneau, E. Axente, G. Coustillier, T. Itina, J. F. Guillemoles and P. Alloncle: J. Phys. D, 2006, 39D, 453-460.

6. S. I. Anisimov, B. L. Kapeliovich and T. L. Perelman: Sov. Phys. Jet., 1974, 39, 375-377.

7. S. Nolte., C. Momma, H. Jacobs, A. Tünnermann, B. N. Chichkov and H. Welling: J. Opt. Soc. Am. B, 1997, 14B, 2716-2722.

8. J. K. Chen and J. E. Beraun: J. Opt. A, Pure Appl. Opt., 2003, 5A, 168-173.

9. L. Jiang and H. Tsai: J. Heat Transfer., 2005, 127, 1167-1173.

10. K. Dou, R. L. Parkhill, J. Wu and E. T. Knobbe: IEEE J. Selected Topic Quantum Electron., 2001, 7, 567-578.

11. B. H. Christensen, K. Vestentoft and P. Balling: Appl. Surf. Sci., 2007, 253, 6347-6352.

12. P. Lechner and H. Schade: Prog. Photovolt. Res. Appl., 2002, 10, $85-97$. 
13. A. Boagaerts, Z. Chen, R. Gijbels and A. Vertes: Spectrochim. Acta Part B, 2003, 58B, 1867-1893.

14. J. L. Ocaña, C. Molpeceres, M. Morales and A. García-Beltrán: SPIE Proc., 2000, 3885, 252-263.

15. Q. Quanming, S. S. Mao, X. Mao and R. E. Russo: Appl. Phys. Lett., 2002, 80, 3072-3074.

16. S. S. Mao, X. L. Mao, R. Greif and R. E. Russo: Appl. Surf. Sci. 1998, 127-129, 206-211.
17. L. Quanming, S. S. Mao, X. Mao and R. E. Russo: Appl. Phys. Let., 2002, 80, 3072-3074.

18. Math Works Incorporation: MATLAB user manual version 7.1 R14, Natick, MA, Math Works Inc., 2005.

19. R. D. Skeel and M. Berzins: SIAM J. Sci. Statist. Comput., 1990, $11,1-32$.

20. M. Weber: 'Handbook of optical materials'; 2003, Boca Raton, FL, CRC Press. 